N. E. Piskunov, W. W. Weiss, D. F. Gray, eds.

\title{
Stellar Abundance Analyses in the Light of 3D Hydrodynamical Model Atmospheres
}

\author{
Martin Asplund \\ Research School of Astronomy and Astrophysics, Mt Stromlo \\ Observatory, Cotter Road, Weston, ACT 2611, Australia
}

\begin{abstract}
.
I describe recent progress in terms of 3D hydrodynamical model atmospheres and 3D line formation and their applications to stellar abundance analyses of late-type stars. Such 3D studies remove the free parameters inherent in classical 1D investigations (mixing length parameters, macro- and microturbulence) yet are highly successful in reproducing a large arsenal of observational constraints such as detailed line shapes and asymmetries. Their potential for abundance analyses is illustrated by discussing the derived oxygen abundances in the Sun and in metal-poor stars, where they seem to resolve long-standing problems as well as significantly alter the inferred conclusions.
\end{abstract}

\section{Introduction}

Determining stellar element abundances play an integral role in most endeavours to improve our understanding of stellar, galactic and cosmic evolution. The term observed abundances is somewhat of a misnomer however, since the chemical composition of course can not be inferred directly from an observed spectrum. The obtained abundances are therefore never more trustworthy than the models employed to analyse the observations. The derivation of accurate abundances require realistic models of both the spectrum formation region and the spectrum formation process, which is the topic of the present contribution.

For a long time, understanding stellar convection has been one of the greatest challenges in stellar astrophysics. Convection can provide an efficient means of transporting energy and thereby greatly influence the stellar structure and evolution, while also mixing nuclear-processed material and preventing radiative diffusion and gravitational settling. For late-type stars, the surface convection zone reaches the stellar atmosphere, which directly affects the emergent spectrum. The solar granulation is the observational manifestation of convection: concentrated, rapid, cold downdrafts in the midst of broad, slow, warm upflows. Qualitatively similar granulation properties are expected in other solartype stars, as indeed confirmed by 3D numerical simulations (e.g. Nordlund \& Dravins 1990; Asplund et al. 1999; Asplund \& García Pérez 2001; Allende Prieto et al. 2002) and indicated by observed line asymmetries. The up- and downflows have radically different temperature structures (Stein \& Nordlund 1998), which can not be approximated by normal theoretical $1 \mathrm{D}$ hydrostatic model at- 
mospheres with different effective temperatures $T_{\text {eff }}$ (Fig. 1). In fact, neither the steep temperature structures of the ascending material nor the shallow gradients of the descending gas are well described by such 1D models. Because of the photospheric inhomogeneities and the highly non-linear and non-local nature of spectrum formation, it is clear that no single $1 \mathrm{D}$ model can be expected to properly describe all aspects of what is inherently a 3D phenomenon.

Here I will describe recent progress in developing 3D hydrodynamical model atmospheres of late-type stars and some applications to stellar abundance analyses. In spite of their still inferior treatment of radiative transfer and line blanketing compared with existing 1D model atmospheres based on the mixing length theory, detailed comparisons with a range of observational diagnostics suggest that these simulations are indeed highly realistic.

\section{3D Hydrodynamical Model Atmospheres}

The 3D model atmospheres which form the basis of the abundance analyses presented here have been computed with a $3 \mathrm{D}$, time-dependent, compressible, explicit, radiative-hydrodynamics code developed to study solar and stellar surface convection (Stein \& Nordlund 1998). The hydrodynamical equations for conservation of mass, momentum and energy are solved on a non-staggered Eulerian mesh with gridsizes of $\approx 100^{3}$. The physical dimensions of the grids are sufficiently large to cover many $(>10)$ granules simultaneously in the horizontal direction and about 13 pressure scale-heights in the vertical. In terms of continuum optical depth the simulations extend at least up to $\log \tau_{\text {Ross }} \approx-5$ which for most purposes are sufficient to avoid numerical artifacts of the open upper boundary on spectral line formation. The lower boundary is located at large depths to ensure that the inflowing gas is isentropic and featureless, while periodic horizontal boundary conditions are employed. The temporal evolution of the simulations cover several convective turn-over time-scales to allow thermal relaxation to be established and to obtain statistically significant average atmospheric structures and spectral line profiles. No magnetic fields have been included in the simulations described here although the code is capable of simulating magneto-convection.

In order to obtain a realistic atmospheric structure, it is crucial to have the best possible input physics, and properly account for the energy exchange between the radiation field and the gas. The adopted equation-of-state is that of Mihalas et al. (1988), which includes the effects of ionization, excitation and dissociation. The continuous opacities come from the Uppsala package (Gustafsson et al. 1975 and subsequent updates) while the line opacities are from Kurucz (1998, private communication). The 3D radiative transfer is solved at each timestep using 9 inclined rays ( $2 \mu$-angles and $4 \varphi$-angles, plus the vertical) under the assumptions of local thermodynamic equilibrium (LTE, $S_{\lambda}=B_{\lambda}$ ) and opacity binning (Nordlund 1982). The four opacity bins are designed to correspond to continuum and weak, intermediate and strong lines. The assignment of the original 2748 wavelength points into the different opacity bins follows from detailed monochromatic radiative transfer calculations of the $1 \mathrm{D}$ averaged atmospheric structure. The opacity binning thus includes the effects of line-blanketing in a manner reminiscent of opacity distribution functions. In order to improve the 


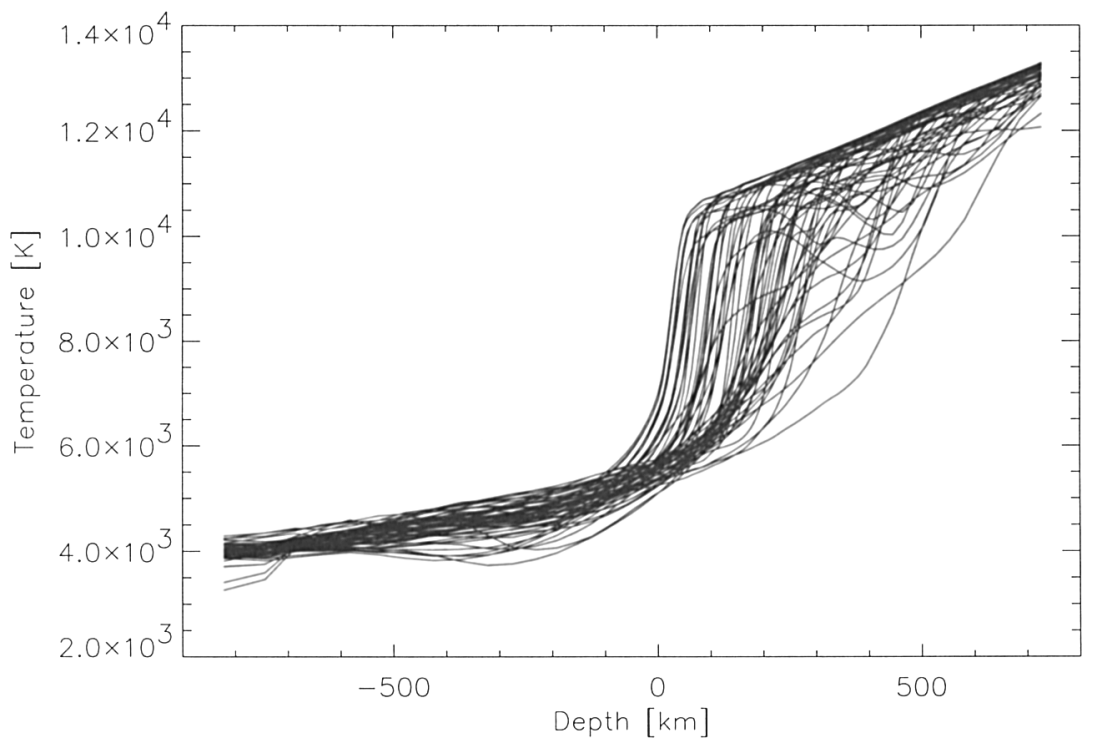

Figure 1. Temperature structure of a 3D solar model atmosphere illustrating the vastly different temperature gradients in the up- and downflows. Vertical columns are connected with solid lines.

numerical accuracy, the radiative transfer is solved on a finer grid and the results subsequently interpolated back to the original grid for the hydrodynamical variables at each timestep.

It is important to realise that the simulations contain no free parameters which are tuned to improve the agreement with observations. The adoption of the numerical and physical dimensions of the simulation box is determined by practical computional time considerations, the need to resolve the most important spatial scales and the wish to place the artificial boundaries as far as possible from the region of interest. The code is stabilized using a hyper-viscosity diffusion algorithm with parameters specified from standard hydrodynamical test cases. These parameters are not changed in the actual stellar convection simulation and are therefore not freely adjustable parameters. It has been verified that the resulting atmospheric structures are insensitive to the adopted effective viscosity at the current highest affordable numerical resolution (Asplund et al. 2000a). The input parameters discriminating different models are the surface gravity $\log g$, metallicity $[\mathrm{Fe} / \mathrm{H}]$ and the entropy of the inflowing material at the bottom boundary. The effective temperature of the simulation is therefore a property which depends on the entropy structure and evolves with time around its mean value following changes in the granulation pattern.

Further details of the 3D hydrodynamical model atmospheres are available in Stein \& Nordlund (1998), Asplund et al. (1999, 2000a,b) and Asplund \& García Pérez (2001). 


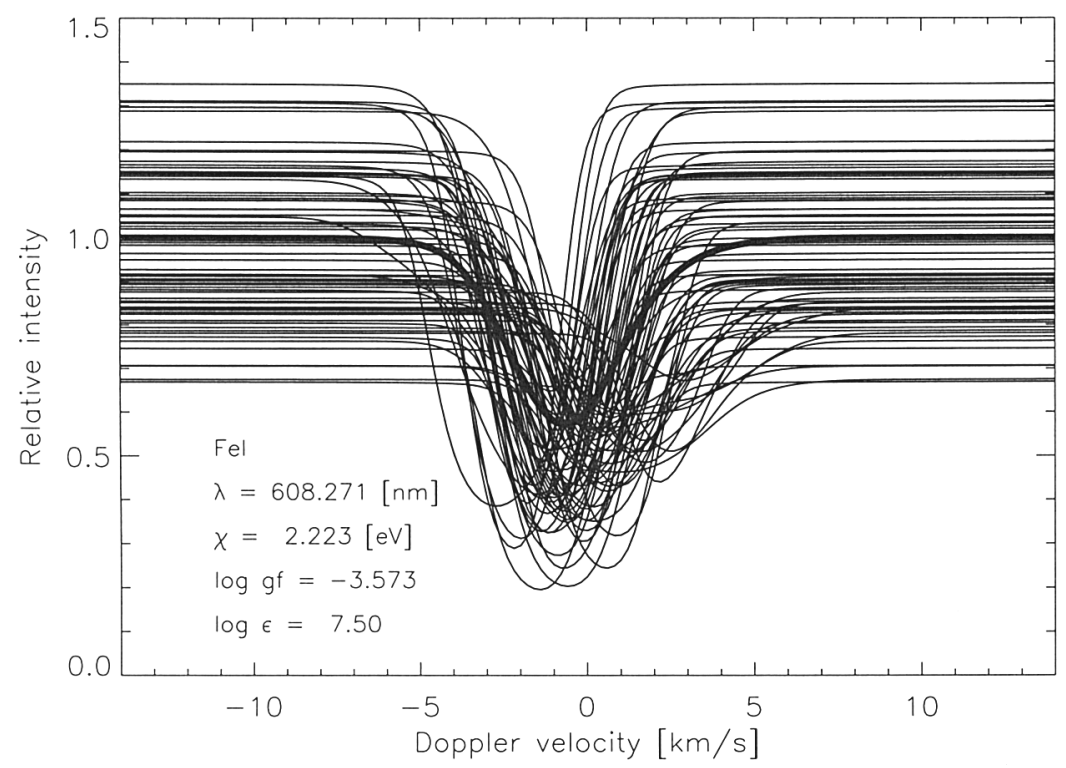

Figure 2. Predicted spatially resolved profiles of the Fe I $608.2 \mathrm{~nm}$ line in the solar simulation. Note the bias of stronger lines in the upflows due to their steeper temperature gradients in general.

\section{Spectral Line Formation}

\subsection{General Description}

The 3D hydrodynamical model atmospheres described in the previous section form the basis for the 3D spectral line formation calculations. The Doppler shifts introduced by the convective and oscillatory motions cause line asymmetries and shifts as well as broaden the lines significantly. Spatially resolved profiles thus come in an amazing assortment as a result of the atmospheric inhomogeneities and velocities together with their correlations (Fig. 2). The concepts of microand macroturbulence, which are introduced in 1D analyses in order to account for the missing line broadening, are not necessary in 3D calculations, as evident from the excellent agreement between predicted and observed line profiles (Fig. 3 , see also Asplund et al. 2000b) and the lack of significant trends in derived abundances with line strength (Asplund et al. 2000c; Asplund 2000). The main component of microturbulence does not arise from microscopic turbulent motions but from gradients in convective motions that are resolved with the present simulations, which is also the main physical explanation for macroturbulence.

The original 3D hydrodynamical simulations which includes deep, extremely optically thick layers are interpolated to a finer depth scale which extends only down to $\log \tau_{\text {Ross }} \approx 2.5$ prior to the spectral line calculations for improved numerical accuracy. At the same time, the simulations are interpolated to a coarser horizontal grid to ease the computational burden. Extensive tests have en- 
sured that this practice is acceptable even when considering fine details such as line asymmetries; for abundance analysis purposes the procedure introduces non-noticable differences $(<0.01$ dex for individual snapshots and much less for temporal averages). Depending on whether the 3D line formation is computed in LTE or in non-LTE, different assumptions and approximations are necessary, which are described next.

\subsection{D LTE Line Formation}

With the assumption of $\operatorname{LTE}\left(S_{\lambda}=B_{\lambda}\right)$, the level population is uniquely determined by the local gas temperature from the Boltzmann and Saha distributions. With the source function known, it is then straightforward to solve the radiative transfer equation, which typically is performed for about 100 wavelength points across the line profile for 10-20 inclined outgoing rays for more than 50 different snapshots. This allows asymmetries and shifts to be determined to an accuracy on the $\mathrm{ms}^{-1}$-level. For abundance determinations even individual snapshots give results accurate to within about $0.03 \mathrm{dex}$, since at any instant many granules are covered by the simulation box. All in all, a single temporally and spatially averaged flux profile in 3D correspond most of the time to $N_{\mathrm{t}} * N_{\mathrm{x}} * N_{\mathrm{y}} * N_{\text {angles }} * N_{\lambda} \gtrsim 10^{8} 1 \mathrm{D}$ radiative transfer calculations. In addition, each $3 \mathrm{D}$ profile is normally computed for at least three different abundances to enable interpolation to the requested line strength. Even then, such 3D LTE line calculations are achievable on current workstations thanks to efficient numerical algorithms and implementations.

No micro- and macroturbulence enter the calculations but for flux profiles the projected stellar rotational velocity $v_{\text {rot }} \sin i$ causes line broadening in addition to the natural, pressure and convective broadening. Flux profiles are obtained after the actual radiative transfer calculations from a disk-integration of the stored profiles of the individual inclined rays for a given $v_{\text {rot }} \sin i$ (Dravins $\&$ Nordlund 1990). The detailed comparison with observations can then proceed through a $\chi^{2}$-analysis by interpolating the computed profiles with varying abundances and adopting different $v_{\text {rot }} \sin i$.

\subsection{D Non-LTE Line Formation}

Recently, even detailed 3D non-LTE line formation calculations have become feasible (Kiselman 1997; Uitenbroek 1998; Botnen \& Carlsson 1999; Asplund et al. 2002). Without the much simplifying assumptions of Saha and Boltzmann distribution for the level population, the rate equations assuming statistical equilibrium $\left(\mathrm{d} n_{\mathrm{i}} / \mathrm{d} t=0\right)$ must be solved simultaneously with the radiative transfer equation. Due to the much increased computational effort in achieving this, sacrifies must be made in other areas, most notably in terms of number of snapshots the calculations can be made for. With individual snapshots it is still feasible to carry out calculations for atoms consisting of hundreds of radiative transitions, each with 50-100 wavelength points. Fortunately, with 3D model atmospheres the non-LTE effects are expected to be quite similar between different snapshots due the relatively large area coverage always containing many up- and downflows, which is also confirmed by detailed calculations. The by far dominating part of 3D non-LTE computations of reasonably sized model atoms is the actual radiative transfer solution from the current estimate of the source 

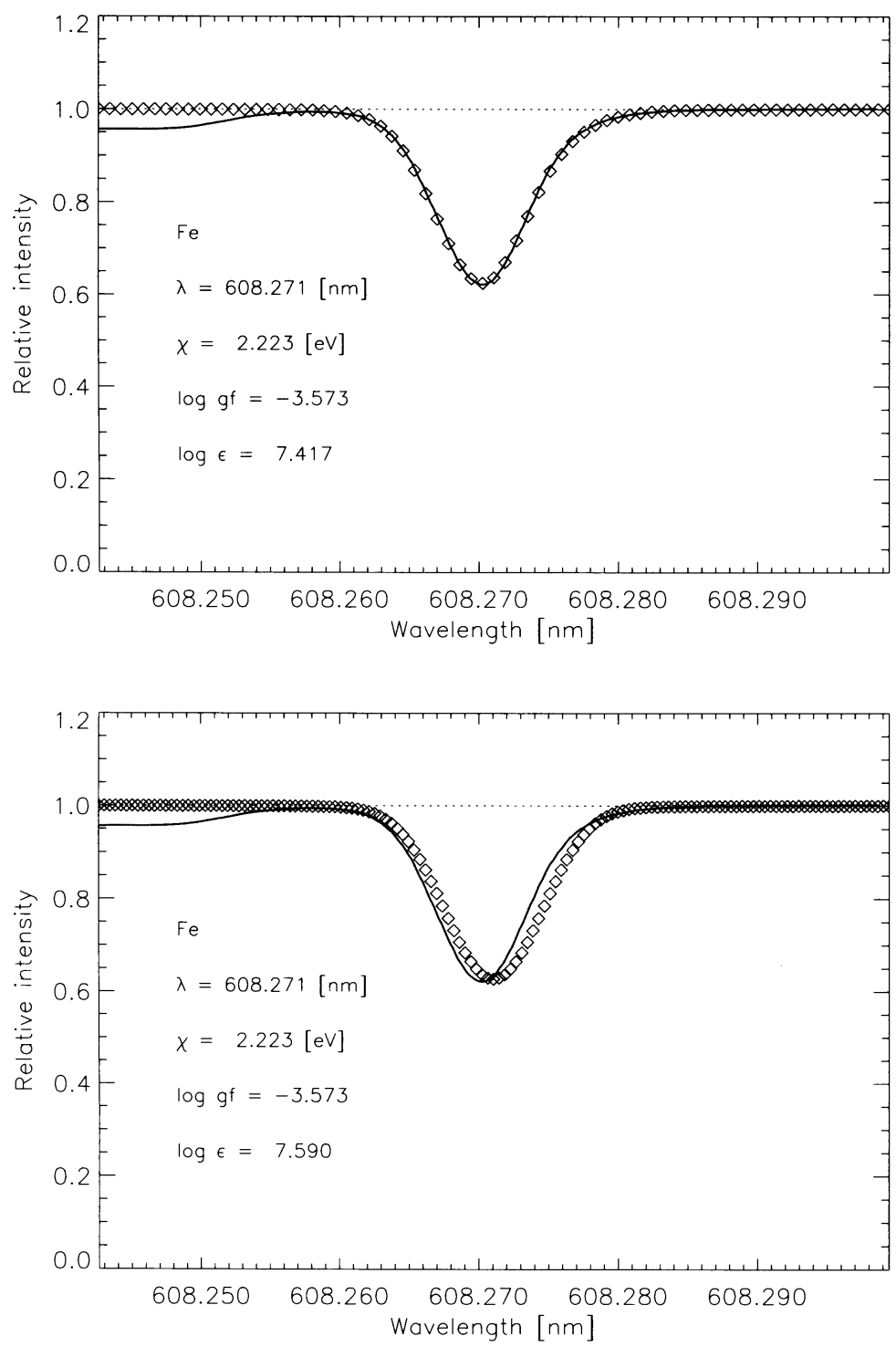

Figure 3. Upper panel: Comparing the predicted temporally and spatially averaged intensity profile of the Fe I $608.2 \mathrm{~nm}$ line (diamonds) with the observed solar profile (solid line). Lower panel: Corresponding 1D profile using the Holweger-Müller model atmosphere with an optimized macroturbulence (Gaussian since radial-tangential macroturbulence is not applicable for intensity profiles) and a microturbulence of $1.0 \mathrm{~km} \mathrm{~s}^{-1}$. Note the inability of the 1D case to predict the line shift and asymmetry, as well as the different required Fe abundance $(+0.17 \mathrm{dex})$ for the same line strength. 
function. This formal solution of the radiative transfer equation is performed for all wavelengths of all transitions for typically 24-48 inclined rays to compute mean intensities and flux profiles. The final converged solution is then obtained by a iterative procedure, normally employing an accelerated lambda iteration technique or similar, allowing convergence typically within 10 iterations. For small model atoms, the calculations are possible to perform on modern workstations but for more extended atoms the use of supercomputers are better suited, not the least due to vast memory requirements with 3D non-LTE codes such as MULTI3D (Botnen \& Carlsson 1999; Asplund et al. 2002).

\section{Stellar Abundance Analyses with 3D Model Atmospheres}

The realistic 3D hydrodynamical model atmospheres of late-type stars together with corresponding LTE and non-LTE line formation tools described above have a natural application to stellar abundance analyses. For abundance studies, the different mean photospheric structure between $3 \mathrm{D}$ and $1 \mathrm{D}$ models, the existence of atmospheric inhomogenities and the broadening of spectral lines due to convective motions are all important ingredients for the line formation. To illustrate the possible impact of the new generation of 3D model atmospheres on abundance analyses, I will describe the situation for the most abundant cosmic metal, oxygen, in two important types of stars: the Sun and metal-poor halo stars. In both cases it seems like the adoption of 3D model atmospheres drastically affects the conclusions.

\subsection{Oxygen in the Sun}

The solar oxygen abundance ought to be well determined but astonishingly the commonly adopted value may be in error by almost a factor of two. For the Sun, five different types of diagnostics are potentially available: permitted high-excitation $\mathrm{O}$ I lines, the forbidden [O I] lines at $630 \mathrm{~nm}$, the $\mathrm{OH}$ vibrationalrotational and pure rotational lines in the IR, and finally the $\mathrm{OH}$ electronic transitions in the UV (which have not been included in the present analysis). All have quite different sensitivities to the atmospheric structure and the line broadening. In addition, all have their pros and cons: the [O I] lines are very weak and likely blended (Allende Prieto et al. 2001); the O I lines are sensitive to departures from LTE (Kiselman 1993); the OH IR lines are, like all molecular features, very temperature sensitive (Asplund \& García Pérez 2001); the $\mathrm{OH}$ UV lines may be affected by missing opacity (Balachandran \& Bell 1998) and possibly non-LTE effects (Asplund \& García Pérez 2001). Unfortunately the different lines imply wildly discordant abundances, the difference amounting to about 0.3 dex both for the Holweger-Müller (1974) and MARCS (Asplund et al. 1997) $1 \mathrm{D}$ model atmospheres. Normally the conclusions from the OH IR have been embraced $\left(\log \epsilon_{\mathrm{O}} \approx 8.90\right)$, largely due to the remarkably small line-to-line scatter (Sauval et al. 1984). An application of our new 3D model atmospheres to the problem, however, suggests that the solar oxygen abundance may be significantly lower $\left(\log \epsilon_{\mathrm{O}} \approx 8.65\right)$ than previously thought (Allende Prieto et al. 2001; Asplund et al., in preparation).

[O I]: Due to the exceptionally good agreement between predicted and observed line profiles in general with $3 \mathrm{D}$ model atmospheres (Fig. 3), disturbing 
blends are readily identified. This statement naturally presumes that detailed comparisons have already been performed for a range of lines with similar properties and typical line formation regions, which fortunately is the case for [O I]. The very poor agreement between the theoretical and the observed profile, immediately signals that the $[\mathrm{OI}] 630.0 \mathrm{~nm}$ line is indeed blended, which have occasionally been suspected but mostly thought to be relatively unimportant. The effect of the blending Ni I line reduces the derived abundance significantly $(-0.13 \mathrm{dex})$, while the transition from 1D to 3D model atmospheres and a revised $g f$-value are of less importance $(-0.08$ and -0.03 dex, respectively) but compounds the overall difference. The forbidden line implies a solar oxygen abundance of $\log \epsilon_{\mathrm{O}}=8.69 \pm 0.05$ (Allende Prieto et al. 2001).

$\mathrm{O}$ I: The $\mathrm{O}$ I triplet around $777 \mathrm{~nm}$ is well-known to be susceptible to significant departures from LTE. We have therefore investigated its line formation in detail with the 3D non-LTE code MULTI3D (Botnen \& Carlsson 1998; Asplund et al. 2002), employing two independent snapshots from the 3D solar simulation and a model atom consisting of 23 levels and 65 radiative transitions (Kiselman 1993). The adopted model atom is sufficiently comprehensive for this particular problem since even a two-level approach catches the essentials of the line formation. Collisional excitation and ionization due to electrons are accounted for but not the corresponding case of hydrogen collisions, since the available classical recipes (Drawin 1968) likely over-estimates their importance by several orders of magnitude. Our 3D non-LTE calculations indeed reveal pronounced departures from LTE, in terms of abundance about $0.2 \mathrm{dex}$. The dominant non-LTE mechanism is the same as with $1 \mathrm{D}$ models, namely photon losses in the line itself. Preliminary the triplet implies a solar oxygen abundance of $\log \epsilon_{\mathrm{O}}=8.67 \pm 0.03$ (Asplund et al., in preparation).

OH vibrational and rotational lines: The slightly cooler temperature structure in our 3D solar model compared with the standard Holweger-Müller (1974), which is normally the 1D model of choice for solar abundance determinations, directly translates to a lower derived oxygen abundance from the $\mathrm{OH}$ IR lines. Equally important, however, is the presence of temperature inhomogeneities due to the non-linear line formation. It therefore comes as no surprise that $1 \mathrm{D}$ analyses typically will significantly over-estimate the abundances derived from molecular lines. We have reanalysed 70 and $127 \mathrm{OH}$ vibrationrotation and pure rotation lines, respectively, which have previously been studied by Grevesse et al. (1984) and Sauval et al. (1984) using up-to-date molecular line data. The 3D line formation calculations are based on the assumption of LTE, which is likely a reasonable approximation in view of the efficient collisional thermalization of the relevant levels. A potential source of error comes from the assumed chemical equilibrium in the $\mathrm{OH}$ molecule formation, which would tend to artificially decrease the oxygen abundance slightly. The magnitude of this effect is not known at this stage but will be investigated further in the near future. Keeping this in mind, preliminary unweighted means yield $\log \epsilon_{\mathrm{O}}=8.61 \pm 0.03$ for the vibrational lines and $\log \epsilon_{\mathrm{O}}=8.67 \pm 0.03$ for the rotational lines.

Summary: Although the exact value for the solar oxygen abundance depends on the relative weights the different diagnostics are given, it is clear that our detailed $3 \mathrm{D}$ investigation unanimously points to a significantly lower solar 

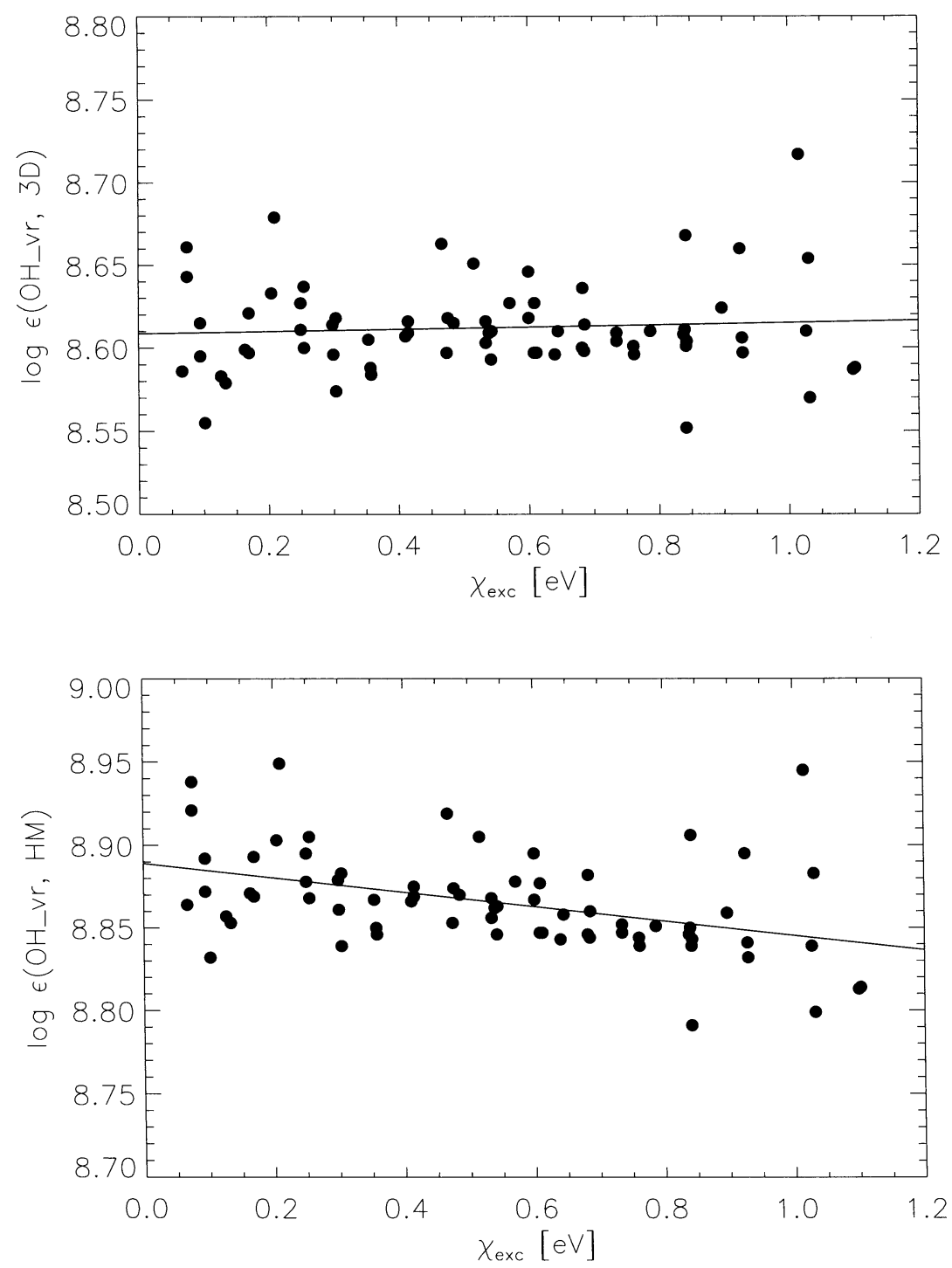

Figure 4. Upper panel: Derived $\mathrm{O}$ abundances from the $\mathrm{OH}$ vibrational-rotational lines in the IR using a 3D hydrodynamical simulation of the solar atmosphere. The solid line denotes the leastsquare-fit to the data. Lower panel: Corresponding results for the 1D Holweger-Müller semi-empirical model atmosphere. Note the distinctly higher derived abundances compared with the 3D case. 
oxygen abundance than previously thought based on classical 1D model atmospheres. Preliminary we estimate $\log \epsilon_{\mathrm{O}}=8.65 \pm 0.05$ which is lower by almost a factor of two. Such a radical re-evaluation for the most abundant metal in the star we know the best is certainly a sobering realisation when discussing derived stellar abundances based on inferior spectra and where the modelling uncertainties can be much larger. Our revision directly impacts also on stellar evolution calculations and helioseismology among other topics.

\subsection{Oxygen in Metal-Poor Stars}

The oxygen abundances in stars born at different epochs and locations carry vital information about the formation and evolution of galaxies. In particular the temporal (with metallicity as a practical although not perfect proxy) evolution of the oxygen-to-iron ratio can yield estimates of the time-scales involved (O mainly from SNe II while Fe largely from SNe Ia) as well as give important clues to the nature of the elusive Pop III stars. It has long been known that halo stars show an over-abundance of oxygen $([\mathrm{O} / \mathrm{Fe}]>0)$ but an exact estimate has been notoriously difficult to achieve due to the large discrepancies between the different abundance indicators. Typically the [O I] line implies a more or less plateau at $[\mathrm{O} / \mathrm{Fe}] \approx+0.4$ (Barbuy 1988; Nissen et al. 2002) but due to weakness of the line its use for $[\mathrm{Fe} / \mathrm{H}]<-2.0$ is restricted to analysis of giants and subgiants. Recent analyses of the $\mathrm{OH}$ UV lines in halo dwarfs on the other hand seem to suggest an almost linear trend with metallicity, reaching $[\mathrm{O} / \mathrm{Fe}] \approx+1.0$ at $[\mathrm{Fe} / \mathrm{H}]=-3.0$ (Israelian et al. 1998; Boesgaard et al. 1999) while the OH IR vibrational lines in giants again more support the conventional plateau (Melendez \& Barbuy 2002). The confusing situation is completed by the fact that the oxygen abundances derived from the high-excitation $\mathrm{O}_{\mathrm{I}}$ triplet at $777 \mathrm{~nm}$ tend to lay in between the $\mathrm{OH} \mathrm{UV}$ and the $[\mathrm{O} \mathrm{I}]$ results.

We have recently applied the new generation of 3D hydrodynamical model atmospheres of metal-poor stars (Asplund et al. 1999) in an attempt to investigate whether a possible resolution to the oxygen conundrum can be found. Due to the lack of radiative heating from spectral lines in halo stars, the adiabatic cooling from the rising gas in the upflows dominate the energy balance. Consequently, the temperature is much below the radiative equilibrium value in the optically thin layers, which is enforced in 1D models.

OH UV: As noted before, molecule formation is extremely temperature sensitive. The lower temperatures in $3 \mathrm{D}$ models therefore directly translates to much larger number densities of molecules and thus to stronger lines. The application of 3D model atmospheres appears to bring down the high advocated linear trend to something which better resembles the canonical plateau (Asplund \& García Pérez 2001), revealing a potential and very significant systematic error in previous standard analyses using 1D model atmospheres. This 3D LTE correction can easily amount to $0.6 \mathrm{dex}$ or more at $[\mathrm{Fe} / \mathrm{H}]=-3.0$. The final word on this topic is not written yet however, as the $3 \mathrm{D} \mathrm{OH}$ analyses sofar make the crucial assumption of LTE both in the line formation and in the $\mathrm{OH}$ molecule formation. Existing non-LTE calculations for molecules are very rare. Asplund \& García Pérez (2001) found the possibility of significant departures from LTE in the $\mathrm{OH}$ line formation but with little metallicity dependence using a very simplified two-level approach in $1 \mathrm{D}$; the $3 \mathrm{D}$ case still remains to be investigated. 
In addition, due to the rapid cooling of the upflowing material it is not unlikely that the time available is not sufficient for equilibrium chemistry to be established, in particular for metal-poor stars with their very low temperatures in the upper layers.

[O I]: One could imagine that the [OI] line should be insensitive to the low surface temperatures in 3D models of halo stars insofar it originates from the ground level of the ionization stage where essentially all oxygen is residing. In reality though there are significant downward 3D corrections also for this line, since the line strength depends on the ratio of line and continuous opacity. The latter is dominated by $\mathrm{H}^{-}$whose number density is proportional to the free electrons which are much reduced in the low temperature gas (Nissen et al. 2002). In terms of abundance the $3 \mathrm{D}$ corrections amount to about $-0.2 \mathrm{dex}$ at $[\mathrm{Fe} / \mathrm{H}] \approx-2.0$ for dwarfs and subgiants. Whether a similar revision is needed for giants have to await the construction of 3D models for such stars but slightly smaller corrections would not be unexpected.

$\mathbf{O}$ I: The most problematic of the available diagnostics in metal-poor stars is likely the O I lines. Due to their high excitation potential, the lines are formed in very deep layers where the differences between $3 \mathrm{D}$ and $1 \mathrm{D}$ models are small. Already in 1D analyses, the triplet yields higher $\mathrm{O}$ abundances than $[\mathrm{O} \mathrm{I}]$ which will be aggrevated unless there are large metallicity-dependent 3D non-LTE effects on the triplet or the adopted $\mathrm{T}_{\text {eff-scale needs significant revision. Pre- }}$ liminary 3D non-LTE calculations for the halo subgiant HD 140283 (Asplund et al., in preparation) suggest quite similar abundance corrections to the solar case, $\approx-0.2 \mathrm{dex}$, which is apparently not enough (Nissen et al. 2002). The best $\mathrm{T}_{\text {eff-scale for halo stars likely comes from the IR flux method, which gives }}$ too low $\mathrm{T}_{\text {eff }}$ to resolve the discrepancy even with $3 \mathrm{D}$ models (Asplund \& García Pérez 2001). The use of 3D models may have resolved the problem between the $[\mathrm{O} I]$ and the OH UV lines but clearly not all answers are in for the O I triplet.

Summary: It seems like the adoption of the new generation of 3D hydrodynamical model atmospheres holds a crucial key to the resolution of the long-standing problem of oxygen abundances in halo stars. Although the final answer has not yet emerged, the existing results together with the recent downward revision of the solar $\mathrm{O}$ abundance described above point to a nearly flat $[\mathrm{O} / \mathrm{Fe}]$ trend but with a slow rise for $[\mathrm{Fe} / \mathrm{H}] \lessgtr-2$, i.e. somewhere in between the hotly contested plateau and linear trends (Nissen et al. 2002).

\section{Concluding Remarks}

The above examples illustrate that existing 1D analyses may well be hampered by significant systematic errors which can severely impact the inferred conclusions. With the advent of a new generation of telescopes and instrumentation it is clear that the dominant source of error is no longer of observational nature but rather stems from uncertainties inherent to the actual analyses. There is obviously a great need for investing also significant resources in improving the modelling aspects in order to place the findings on firmer footing. It is noteworthy that the main obstacle for achieving this is simply a lack of manpower, in spite of the existence of a very large community of astronomers relying on such model atmospheres for their applications. 
Acknowledgments. The work described in this contribution represent the results of successful collaborations with a large number of wonderful colleagues, in particular Carlos Allende Prieto, Mats Carlsson, Remo Collet, Ana García Pérez, Nicolas Grevesse, Dan Kiselman, David Lambert, Poul Erik Nissen, Åke Nordlund, Francesca Primas, Jacques Sauval, Bob Stein, and Regner Trampedach. Finally, I am grateful to the editors for their patience.

\section{Discussion}

BORRERO: FTS data used to compare synthetic profiles are affected by quiet sun magnetic fields making the spectral lines to broad. How can this affect abundance determinations by using 3D-hydrodynamical model where magnetic field is neglected?

ASPLUND: Spectral line formation is heavily biased toward the upflows which have large area coverage, higher continuum intensities and steeper temperature gradients, all combining to produce stronger lines. Such upflows have very weak magnetic fields due to the diverging nature of the flow. Therefore the neglect of magnetic fields should not be very crucial for the quiet Sun. In addition we have on purpose avoided lines known to be magnetically sensitive so the missing line broadening should be minimal. Finally, we normally try to use weak lines unaffected by broadening, which makes the derived abundances more secure. However, our simulations can naturally not be employed for very magnetically active stars where the magnetic fields influence both the atmosphere structure and line formation.

KURUCZ: Isn't there a logical inconsistency between using 3D models that do not have realistic opacity, realistic radiation treatment, or realistic equation of state and making sophisticated non-LTE or molecular calculations that require all three?

ASPLUND: Our 3D hydrodynamic model atmospheres do indeed include realistic input physics in terms of opacities, equation - of - state and radiative transfer. In particular we are using the same opacities that go in to the widely used ID MARCS models, including all line opacities from the Kurucz database, while we adopt the Mihalas-Hummer-Däppen equation-of-state. The radiative transfer accounts for line-blanketing using opacity binning techniques (similar to having one giant opacity distribution function). Naturally there is always room for improvement, however, the accuracy of the opacity binning is regularly checked during the simulations by solving the monochromatic case with thousands of wavelengths and always found to agree to within $1 \%$ in terms of total radiative heating/cooling, although we have a much more simplified radiative transfer for convection, which appears to be a much more important ingredient for late-type stars. We are however currently working on improving the radiative transfer further with promising preliminary results.

TITLE: Don't forget, that magnetic fields have effects that are not localized to the field regions alone. At solar maximum 10-20\% of the sun may have abnormal granulation here both up and down flows are significantly reduced. 
ASPLUND: The simulations I presented do not include magnetic fields and thus can not represent magnetically active stars. Bob Stein and Åke Nordlund have recently started working on magneto-convection using the same code, which we will eventually use also for spectral line formation studies. It is certainly true that magnetic fields for stars such as the Sun at activity maximum could influence the emergent flux. However, there may be factors, which limit their influence: Spectral line formation is heavily biased towards the upflows (larger area coverage, larger continuum intensity, steeper temperature gradients) where magnetic fields are very weak ever at activity maximum due to the diverging flow of upflows. This hypothesis should of course be carefully checked with magneto-convection simulations.

\section{References}

Allende Prieto, C., Lambert, D.L., Asplund, M. 2002, ApJ, 556, L65

Allende Prieto, C., Asplund, M., García López, R.J., Lambert, D.L. 2002, ApJ, 567,544

Asplund, M. 2000, A\&A, 359, 755

Asplund, M., García Pérez, A.E. 2001, A\&A, 372, 601

Asplund, M., Carlsson, M., Botnen, A.V. 2002, submitted to ApJ

Asplund, M., Gustafsson, B., Kiselman, D., Eriksson, K. 1997, A\&A, 318, 521

Asplund, M., Nordlund, Å., Trampedach, R., Stein, R.F. 1999, A\&A, 346, L17

Asplund, M., Ludwig, H.-G., Nordlund, Å., Stein, R.F. 2000a, A\&A, 359, 669

Asplund, M., Nordlund, Å., Trampedach, R., Allende Prieto, C., Stein, R.F. 2000b, A\&A, 359, 729

Asplund, M., Nordlund, Å., Trampedach, R., Stein, R.F. 2000c, A\&A, 359, 743

Balachandran, S.C., Bell, R.A. 1998, Nature, 392, 23

Barbuy, B. 1988, A\&A, 191, 121

Boesgaard, A.M., King, J.R., Deliyannis, C.P., Vogt, S. 1999 AJ, 117, 492

Botnen, A.V., \& Carlsson, M. 1999, in Numerical astrophysics, ed. S.M. Miyama et al., 379

Drawin, H.W. 1968, Z. Physik, 211, 404

Grevesse, N., Sauval, A.J., van Dishoek, E.F. 1984, A\&A, 141, 10

Gustafsson, B., Bell, R.A., Eriksson, K., Nordlund, Å. 1975, ApJ, 42, 407

Holweger, H., Müller, E.A. 1974, Solar Physics, 39, 19

Israelian, G., García López, R.J., Rebolo, R. 1998, ApJ, 507, 805

Kiselman, D. 1993, A\&A, 275, 269

Kiselman, D. 1997, ApJ, 489, L107

Meléndez, J., Barbuy, B. 2002, ApJ, 575, 474

Mihalas, D., Däppen, W., Hummer, D.G. 1988, ApJ, 331, 815

Nissen, P.E., Primas, F., Asplund, M., Lambert, D.L. 2002, A\&A, 390, 235

Nordlund, Å. 1982, A\&A, 107, 1

Nordlund, Å., Dravins, D. 1990, A\&A, 228, 155 
Sauval, A.J., Grevesse, N., Brault, J.W., Stokes, G.M., Zander, R. 1984, ApJ, 282,330

Stein, R.F., Nordlund, Å. 1998, ApJ, 499, 914

Uitenbroek, H. 1998, ApJ, 498, 427 